\title{
THE SUCCESSIVE ITERATES OF THE STIELTJES
}

KERNEL EXPRESSED IN TERMS OF THE ELEMENTARY FUNCTIONS*

BY D. V. WIDDER

1. Introduction. The Stieltjes kernel is the function

$$
H_{1}(\xi, \eta)=\frac{1}{\xi+\eta}
$$

We define its successive iterates by the recurrence relation

$$
H_{n}(\xi, \eta)=\int_{0}^{\infty} \frac{H_{n-1}(t, \eta) d t}{\xi+t}, \quad(n=2,3,4, \cdots) .
$$

That these integrals all exist will appear from later considerations. Simple computation shows that

$$
H_{2}(\xi, \eta)=\int_{0}^{\infty} \frac{d t}{(\xi+t)(t+\eta)}=\frac{\log \xi-\log \eta}{\xi-\eta}, \quad(\xi>0, \eta>0) .
$$

It is natural to inquire if it is also possible to express the higher iterates in terms of the elementary functions. It is the purpose of the present note to prove that this is the case. We show, in fact, that $H_{n}(\xi, \eta)$ is a linear combination of the functions

$$
\frac{(\log \xi-\log \eta)^{2 k+1}}{(2 k+1) !(\xi-\eta)}, \quad \frac{(\log \xi-\log \eta)^{2 k}}{(2 k) !(\xi+\eta)}, \quad(k=0,1,2, \cdots),
$$

the constants of combination being the coefficients of the power series expansion of $(\pi / \sin \pi s)^{n}$. The precise result to be proved is contained in Theorem 2 of this paper, stated as follows:

Theorem 2. If $0<\xi<\infty, 0<\eta<\infty$, then

$$
\begin{aligned}
H_{2 n}(\xi, \eta) & =\sum_{k=1}^{n} \frac{A_{2 n, 2 k}}{(2 k-1) !} \frac{[\log \xi-\log \eta]^{2 k-1}}{\xi-\eta},(n=1,2, \cdots), \\
H_{2 n+1}(\xi, \eta) & =\sum_{k=0}^{n} \frac{A_{2 n+1,2 k+1}}{(2 k) !} \frac{[\log \xi-\log \eta]^{2 k}}{\xi+\eta},
\end{aligned}
$$

* Presented to the Society, September 10, 1937. 
where the constants $A_{n, n-k}$ are defined by the expansion

$$
\left(\frac{\pi s}{\sin \pi s}\right)^{n}=A_{n, n}+A_{n, n-1} s+A_{n, n-2} s^{2}+\cdots \text {. }
$$

2. Iterates of the Stieltjes Kernel. We introduce the successive convolutions of the function $\operatorname{sech}(t / 2)$ as follows:

$$
h_{1}(x)=\operatorname{sech}(x / 2) \text {, }
$$

$$
h_{n}(x)=(2 \pi)^{-1 / 2} \int_{-\infty}^{\infty} h_{1}(t) h_{n-1}(x-t) d t, \quad(n=2,3, \cdots) .
$$

Since $h_{1}(x)$ is an even function of class $L$, it follows that $h_{n}(x)$ has the same property. The existence of the successive integrals is obvious.

We are able to express the function $H_{n}(\xi, \eta)$ in terms of $h_{n}(x)$ as follows:

$$
H_{n}\left(e^{x}, e^{y}\right)=(2 \pi)^{(n-1) / 2} 2^{-n} e^{-(x+y) / 2} h_{n}(x-y) .
$$

It is a simple matter of verification to check this result for $n=1$. To prove it by induction, first assume it true for $n-1$. Then

$$
\begin{aligned}
H_{n}\left(e^{x}, e^{y}\right) & =\int_{-\infty}^{\infty} \frac{e^{u} H_{n-1}\left(e^{u}, e^{y}\right)}{e^{x}+e^{u}} d u \\
& =\frac{(2 \pi)^{(n-2) / 2}}{2^{n-1}} \int_{-\infty}^{\infty} \frac{e^{(u-y) / 2} h_{n-1}(u-y)}{e^{x}+e^{u}} d u \\
& =\frac{(2 \pi)^{(n-2) / 2}}{2^{n} e^{(x+y) / 2}} \int_{-\infty}^{\infty} \operatorname{sech} \frac{x-u}{2} h_{n-1}(u-y) d u \\
& =(2 \pi)^{(n-2) / 2} 2^{-n} e^{-(x+y) / 2} \int_{-\infty}^{\infty} h_{1}(t) h_{n-1}(x-y-t) d t \\
& =(2 \pi)^{(n-1) / 2} 2^{-n} e^{-(x+y) / 2} h_{n}(x-y) .
\end{aligned}
$$

This completes the induction.

3. Several Lemmas. In order to establish our principal result we first state several lemmas.

Lemma 1. For all real $x$

$$
\begin{aligned}
(2 \pi)^{-1 / 2} \int_{-\infty}^{\infty} e^{i x t} h_{1}(t) d t & =(2 \pi)^{-1 / 2} \int_{-\infty}^{\infty} e^{i x t} \operatorname{sech}(t / 2) d t \\
& =(2 \pi)^{1 / 2} \operatorname{sech} \pi x
\end{aligned}
$$


This is a familiar result.*

Lemma 2. For all real $x$

$$
(2 \pi)^{-1 / 2} \int_{-\infty}^{\infty} e^{i x t} h_{n}(t) d t=(2 \pi)^{-n / 2}(2 \pi \operatorname{sech} \pi x)^{n} .
$$

This follows from the known facts concerning the Fourier transform of the convolution of several functions.

Lemma 3. If $0<x<1, m=1,2,3, \cdots$, then

$$
\int_{-\infty}^{\infty} e^{-x t} \frac{t^{m}}{1-e^{-t}} d t=\sum_{k=-\infty}^{\infty} \frac{m !}{(x+k)^{m+1}} .
$$

The integral on the left converges absolutely if $0<x<1$ since

for every positive $\epsilon$. Then

$$
\begin{aligned}
\frac{t^{m}}{1-e^{-t}} & =O\left(e^{t t}\right), & & (t \rightarrow \infty), \\
& =O\left(e^{t-\epsilon t}\right), & & (t \rightarrow-\infty),
\end{aligned}
$$

$$
\begin{aligned}
\int_{0}^{\infty} \frac{e^{-x t} t^{m}}{1-e^{-t}} d t & =\int_{0}^{\infty} e^{-x t} t^{m} \sum_{k=0}^{\infty} e^{-k t} d t=\sum_{k=0}^{\infty} \frac{m !}{(x+k)^{m+1}}, \\
\int_{-\infty}^{0} \frac{e^{-x t} t^{m}}{1-e^{-t}} d t & =(-1)^{m} \int_{0}^{\infty} \frac{e^{x t} t^{m}}{1-e^{t}} d t \\
& =(-1)^{m+1} \sum_{k=1}^{\infty} \int_{0}^{\infty} e^{(x-k) t} t^{m} d t=\sum_{k=-\infty}^{-1} \frac{m !}{(x+k)^{m+1}} .
\end{aligned}
$$

The term by term integration is justified by dominated convergence.

Lemma 4. If $-\infty<t<\infty$ and $m=1,2,3, \cdots$, then

$$
\frac{t^{m}}{1-e^{-t}}=\frac{1}{2 \pi i} \int_{1 / 2-i \infty}^{1 / 2+i \infty} e^{s t} \sum_{k=-\infty}^{\infty} \frac{m !}{(s+k)^{m+1}} d s .
$$

This follows from Lemma 3 by a general inversion formula for the Laplace transform. $\dagger$

* See, for example, R. E. A. C. Paley and N. Wiener, Fcurier Transforms in the Complex Domain, 1934, p. 41.

$\dagger$ See, for example, S. Bochner, Vorlesungen über Fouriersche Integrale, Leipzig, 1932, p. 148. 
Lemma 5. If $0<x<1, m=0,1,2, \cdots$, then

$$
\int_{-\infty}^{\infty} \frac{e^{-x t} t^{m}}{1+e^{-t}} d t=\sum_{k=-\infty}^{\infty} \frac{(-1)^{k} m !}{(x+k)^{m+1}}=\lim _{N \rightarrow \infty} \sum_{k=-N}^{N} \frac{(-1)^{k} m !}{(x+k)^{m+1}} .
$$

If $m$ is positive the proof is similar to that of Lemma 3 and is omitted. If $m=0$,

$$
\begin{aligned}
\int_{-\infty}^{\infty} \frac{e^{-x t}}{1+e^{-t}} d t= & \int_{0}^{\infty} e^{-x t} \lim _{N \rightarrow \infty} \sum_{k=0}^{N}(-1)^{k} e^{-k t} d t \\
& -\int_{0}^{\infty} e^{x t} \lim _{N \rightarrow \infty} \sum_{k=1}^{N}(-1)^{k} e^{-k t} d t \\
& =\frac{1}{x}-\int_{0}^{\infty}\left[e^{-x t}-e^{x t}\right] \lim _{N \rightarrow \infty} e^{-t} \frac{\left[1-(-1)^{N} e^{-N}\right]}{1+e^{-t}} d t \\
& =\lim _{N \rightarrow \infty} \sum_{k=-N}^{N} \frac{(-1)^{k}}{x+k} .
\end{aligned}
$$

Since

$$
\begin{aligned}
\mid e^{-t}\left(e^{-x t}-e^{x t}\right)(1- & \left.(-1)^{N} e^{-N t}\right)\left(1+e^{-t}\right)^{-1} \mid \\
& \leqq 2 e^{-t}\left(e^{-x t}+e^{x t}\right)\left(1+e^{-t}\right)^{-1},(0 \leqq t<\infty),
\end{aligned}
$$

and since the right-hand side of this inequality is integrable in the interval $(0, \infty)$, the interchange of limit and integration signs is justified.

Lemma 6. If $-\infty<t<\infty, m=0,1,2, \cdots$, then

$$
\frac{t^{m}}{1+e^{-t}}=\frac{1}{2 \pi i} \int_{1 / 2-i \infty}^{1 / 2+i \infty} e^{s t} \lim _{N \rightarrow \infty} \sum_{k=-N}^{N} \frac{(-1)^{k} m !}{(s+k)^{m+1}} d s .
$$

The proof is the same as that of Lemma 5.

4. Evaluation of the Successive Iterates. We shall first evaluate the functions $h_{n}(x)$ in terms of the elementary functions. The result is contained in the following theorem:

ThEOREM 1. For all real $x$ the functions $h_{n}(x)$ defined by (2) have the expression

$$
\begin{array}{r}
h_{2 n}(x)=\left(\frac{2}{\pi}\right)^{(2 n-1) / 2} \operatorname{csch} \frac{x}{2} \sum_{k=1}^{n} A_{2 n, 2 k} \frac{x^{2 k-1}}{(2 k-1) !} \\
(n=1,2,3, \cdots),
\end{array}
$$


(5) $h_{2 n+1}(x)=\left(\frac{2}{\pi}\right)^{n} \operatorname{sech} \frac{x}{2} \sum_{k=0}^{n} A_{2 n+1,2 k+1} \frac{x^{2 k}}{(2 k) !}$,

$$
(n=0,1,2, \cdots) \text {. }
$$

By use of Lemma 2 and by the known reciprocal formulas for the Fourier transform* we have

$$
h_{n}(x)=(2 \pi)^{-1 / 2} \int_{-\infty}^{\infty} e^{-i x t}(2 \pi)^{n / 2}(\operatorname{sech} \pi t)^{n} d t,(-\infty<x<\infty) .
$$

By the change of variable $s=(1 / 2)-i t$ this becomes

$$
\begin{aligned}
h_{n}(x)=-i(2 \pi)^{(n-1) / 2} e^{-x / 2} \int_{1 / 2-i \infty}^{1 / 2+i \infty} e^{x s}(\csc \pi s)^{n} d s, & \\
& (-\infty<x<\infty) .
\end{aligned}
$$

Now if the constants $A_{n, n-k}$ are defined by (1), it is clear that

$$
\begin{aligned}
& A_{2 n, 2 k-1}=0, \quad(k=1,2, \cdots, n ; n=1,2, \cdots), \\
& A_{2 n+1,2 k}=0, \quad(k=1,2, \cdots, n ; n=0,1,2, \cdots) .
\end{aligned}
$$

The Mittag-Leffler theorem gives us

$$
\begin{aligned}
(\pi \csc \pi s)^{n}=\sum_{k=-\infty}^{\infty}(-1)^{n k}\left[\frac{A_{n, n}}{(s+k)^{n}}+\right. & \frac{A_{n, n-1}}{(s+k)^{n-1}} \\
& \left.+\cdots+\frac{A_{n, 1}}{s+k}\right],
\end{aligned}
$$

with the understanding that

$$
\sum_{k=-\infty}^{\infty} \frac{A_{n, 1}}{s+k}=\lim _{N \rightarrow \infty} \sum_{k=-N}^{N}(-1)^{k} \frac{A_{n, 1}}{s+k} .
$$

We now obtain (4) by substitution of (7) in (6) and by applying Lemma 4. In a similar way (5) is obtained by use of Lemma 6. Theorem 2 now follows from Theorem 1 by virtue of (3).

HARVARD UNIVERSITY

\footnotetext{
* One may apply, for example, S. Bochner, op. cit., Theorem 12, p. 42.
} 